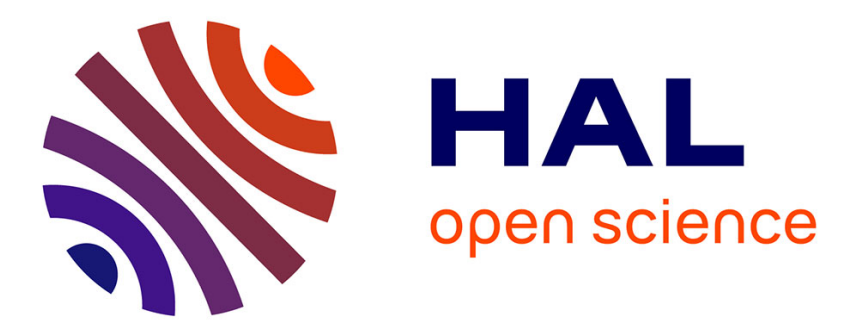

\title{
Isoenzymes d'une population d'Acyrthosiphon pisum Harr (Hom., Aphididae)
}

Antoine Dalmasso, René Bournoville, Michel Bongiovanni, Jean-Marc Bride

\section{To cite this version:}

Antoine Dalmasso, René Bournoville, Michel Bongiovanni, Jean-Marc Bride. Isoenzymes d'une population d'Acyrthosiphon pisum Harr (Hom., Aphididae). Agronomie, 1983, 3 (9), pp.891-896. hal00884586

\section{HAL Id: hal-00884586 https://hal.science/hal-00884586}

Submitted on 1 Jan 1983

HAL is a multi-disciplinary open access archive for the deposit and dissemination of scientific research documents, whether they are published or not. The documents may come from teaching and research institutions in France or abroad, or from public or private research centers.
L'archive ouverte pluridisciplinaire HAL, est destinée au dépôt et à la diffusion de documents scientifiques de niveau recherche, publiés ou non, émanant des établissements d'enseignement et de recherche français ou étrangers, des laboratoires publics ou privés. 


\title{
Isoenzymes d'une population d'Acyrthosiphon pisum Harr (Hom., Aphididae).
}

\author{
Antoine DALMASSO \& René BOURNOVILLE (*) \\ avec la collaboration technique de Michel BongIOVANNI \& Jean-Marc BRIDE \\ I.N.R.A., Station de Recherches sur les Nématodes, 123, Bd Francis-Meilland, F06602 Antibes \\ (*) I.N.R.A., Laboratoire d'Ethologie et d'Ecologie des Insectes, F 86600 Lusignan
}

L'étude d'une population d'Acyrthosiphon pisum, clonée à partir d'œufs d'hiver et de femelles anholocycliques, a montré une variabilité isoenzymatique réduite. Seule une des estérases se caractérisant par un triplet de bandes a présenté une certaine variabilité allélique. Deux autres loci estérasiques, se situant de part et d'autre sur le gel, étaient monomorphes, ainsi que les deux phosphatases et une des deux malate déshydrogénases mises en évidence. Les $\beta$-glucosidase, $\beta$-galactosidase. $\beta$-glucuronidase et $\mathrm{N}$-acétylglucosaminidasc, observées sur gel à gradient, étaient chacune représentécs par unc seule bande, constante d'un clone ou d'un individu à l'autrc. On a pu observer dans les mêmes conditions 4 bandes pour l' $\alpha$ galactosidase et 6 bandes pour les $\alpha$-glucosidases, mais la nécessité d'opérer dans ce cas sur un échantillon global de 100 puccrons n'a pas permis de savoir s'il existait une certaine variabilité individuelle.

Ainsi, l'étude isoenzymatique a fourni peu d'informations susceptibles d'expliquer les différences de comportement parfois observées chez cette espèce au niveau infraspécifique.

Mots clés additionnels : Puceron, estérases, phosphatases, malate déshydrogénase, glycosidases.

Isozymes of a population of Acyrthosiphon pisum (Aphididae).

A population of Acyrthosiphon pisum cloned from overwintering eggs and agamic females was studicd for isozyme polymorphism. Only one esterase, characterized by a triple band, was found to be variable. Two other esterases, two phosphatases and one of the two malate dehydrogenases were monomorphic. $\beta$ glucosidase, $\beta$-galactosidase, $\beta$-glucuronidase and $\mathrm{N}$-acetyl-glucosaminidase observed on gradient gels each showed a constant single band. In the same conditions, there were $4 \alpha$-galactosidase and $6 \alpha$-glucosidase bands. As 100 aphids per sample were used to analyse glycosidase enzymes, it was impossible to know whether these $\alpha$-galactosidases and $\alpha$-glucosidases were polymorphic.

According to these results, the biochemical polymorphism approach could hardly explain the differences in behaviour at intraspecific level often observed in this species.

Addition key-words : Aphid, esterases, phosphatases, malate dehydrogenase, glycosidases.

\section{INTRODUCTION}

L'étude des isoenzymes des pucerons a débuté il y a à peine une dizaine d'années avec les travaux de SUDDERUDDIN (1972), puis de BERANEK (1974) sur Aphis fabae Scop. et aussi sur des clones de Myzus persicae Sulz. résistants aux organophosphorés.

Ces 2 espèces présentent des variations quantitatives importantes pour certaines estérases. Chez $M$. persicae, la résistance aux organophosphorés est directement corrélée à l'abondance de l'estérase $\mathrm{E}_{4}$ (BAKER, 1977; SAWICKI et al., 1978). BUNTING \& VAN EMDEN (1980) ont montré qu'une seule femelle peut produire des descendants diffé- rant par l'activité de cette estérase. Or, cette variabilité ne semble pas résulter de recombinaisons chromosomiques. L'hypothèse avancée est celle d'une amplification au niveau de ce locus, c'est-à-dire que ce gène serait présent en de multiples copies.

Parallèlement à ces travaux, Woll et al. (1978), MAY \& HOLBROOK (1978), prospectant par électrophorèse la diversité génétique du même $M$. persicae, n'ont trouvé que très peu de variabilité isoenzymatique. A l'opposé, TAKADA (1979) a observé 28 formes différant, soit par certaines estérases, soit par leur coloration sur 153 clones analysés.

D'autres travaux ont porté sur la différenciation taxonomique des aphides (SingH \& CunNinghaM, 1981) ou 
sur des modifications des isoenzymogrammes sur des œufs en levée de diapause (LAPCHIN et al., 1981).

SUOMALAINEN et al. (1980), TOMIUK \& WÖHRMANN (1980), SIMON et al. (1982) se sont intéressés à la diversité isoenzymatique interclonale chez diverses espèces, dont précisément Acyrthosiphon pisum Harr. Chez cette espèce, ils ont trouvé des différences sur la malate déshydrogénase $(\mathrm{MDH}), \alpha$-glycérophosphate déshydrogénase ( $\alpha$ GPDH), phosphoglucose isomérase (PGI) et superoxide dismutase (SOD).

L'ensemble des données disponibles donne, en définitive, l'impression qu'il existe une grande similarité dans la structure de phénotypes protéiniques, observés d'une espèce de puceron à l'autre, accompagnée d'une faible variabilité intraspécifique (peu de loci polymorphes et peu d'allèles pour ces loci). La seule exception de la littérature sur ce point est représentée par le travail de TAKADA cité ci-dessus.

Contrastant: avec cette relative uniformité et stabilité isoenzymatiques, le comportement dans les populations d'aphides apparaît assez souvent hétérogène, en particulier lorsqu'il y a confrontation avec un hôte déterminé.

On a ainsi remarqué que la multiplication d' $A$. pisum s'effectue plus ou moins bien selon les populations et les hôtes considérés: pois ou luzerne (BouRnoville, 1977).

Les relations de spécificité entre les insectes et leurs hôtes végétaux dépassent de plus en plus le cadre strictement taxonomique et traditionnel de l'espèce pour se poser en terme de génétique au niveau infraspécifique. Dans un premier temps, on a tendance à prolonger la méthodologie taxonomique à ce niveau en essayant de définir les observations sur les relations plantes ravageurs par des terminologies également classiques : races, biotypes, ... Cette opération essentiellement descriptive des phénomènes n'est porteuse d'aucune explication à leur encontre.

Les analyses qui suivent se proposaient d'apprécier le polymorphisme biochimique sur un certain nombre d'isoenzymes en partant d'un échantillon prélevé dans une population naturelle et de rechercher, ultérieurement, d'éventuelles corrélations entre certains traits du comportement et les phénotypes révélés par les isoenzymogrammes.

\section{ORIGINE DU MATÉRIEL}

Les insectes utilisés ont été prélevés en début d'année, à un moment où on peut différencier l'origine des pucerons.

Deux lots d' $A$. pisum provenant de la Vienne ont fait l'objet de cette étude. Pour le $1^{\mathrm{er}}$ lot, il s'agissait de clones isolés à partir d'une population naturelle composées d'œufs d'hiver et de femelles anholocycliques prélevés dans un champ de luzerne, le 8 février 1981. Le $2^{c}$ lot est également constitué de clones issus de femelles anholocycliques et de fondatrices prélevées le 10 février 1982 dans des caissettes de luzerne infestée à l'automne par $A$. pisum et qui ont passé l'hiver à l'extérieur. La distinction entre les formes holocycliques et les formes anholocycliques a été réalisée par l'observation du stade adulte de chacun de ces clones qui ont été maintenus dans des cages séparćes.

Aucune différence fondamentale n'est résultée de l'interprétation en fonction de ces 2 origines. Ces divers clones ont par ailleurs fait l'objet d'élevages et d'expériences ayant pour but de comparer leur fécondité et d'évaluer, en conditions coritrôlées, leurs possibilités de passage sur une légumineuse annuelle, le pois. Les résultats de ces travaux, qui ne rentrent pas dans le cadre de cette publication, ne fournissent pas de différences importantes entre les clones, ni pour la possibilité de transfert d'hôte, ni pour la comparaison des fécondités lors des 4 premières générations annuelles.

\section{TECHNIQUES}

Les électrophorèses ont été conduites verticalement sur des gels d'acrylamide en plaques de $100 \mu$ ou de $400 \mu$ d'épaisseur permettant l'analyse à partir de l'extrait d'un seul puceron; les types de gel et les conditions d'extraction et de révélation sont portés dans le tableau 1.

Plusieurs tampons d'extraction ont été essayés. Pour les enzymes membranaires ou ceux présents en très petite quantité, nous avons utilisé le Triton X 100 à 0,5 p. 100, qui facilite la libération des molécules enzymatiques.

Pour chaque enzyme étudié nous avons aussi essayé plusieurs types de gels en faisant successivement varier, soit les pourcentages d'acrylamide comme indiqué dans MAURER (1971), soit le $\mathrm{pH}$, en changeant la proportion de Tris(hydroxyméthyl)-aminométhane dans le tampon du gel.

Les glycosidases n'ont pu être obtenues sous forme de bandes bien distinctes qu'avec des gels à gradient et en utilisant une centaine d'individus et non un seul comme pour les autres enzymes. Afin d'accroître nos chances de déceler la variabilité cachée, nous avons utilisé l'isofocalisation pour les phosphatases.

Les techniques de révélation sont, à quelques détails près, celles données par SHAw \& PRASAD (1969).

Les conditions de révélation, sans recours à des inhibiteurs spécifiques, ne permettent pas de préciser la nature exacte des estérases révélées, mais l'objet du travail étant surtout de déceler de possibles variations sur les isoenzymogrammes, nous n'avons pas jugé nécessaire de pousser plus avant l'analyse.

\section{RÉSULTATS}

\section{A. Estérases}

Dans les conditions énoncées, tous les clones d'A. pisum ont distinctement donné 5 bandes se répartissant en 3 zones (probablement 3 loci) (fig. 1). Simon et al. (1982) ont obtenu 4 bandes principales si l'on excepte la bande située à proximité immédiate du dépôt de l'échantillon. Comme ils ont en partie opéré avec du matériel provenant de la Vienne, voisin de celui utilisé ici, on peut penser que la différence est surtout due aux conditions respectives des gels et aux quantités de protéines mises en jeu. Les auteurs signalent une variation quantitative sur la $3^{c}$ estérase, alors que nous avons observé une variation essentiellement qualitative portant sur le "groupe 2 ", représenté dans le cas présent par 3 bandes (fig. 2). Il n'est pas à écarter que la $3^{\text {e }}$ estérase signalée par SIMON et al. (1982) corresponde en fait, pour de simples raisons techniques, au $2^{c}$ groupe d'estérases. Les 3 bandes constituant "ce groupe 2 " se déplacent de façon concomitante d'un individu à l'autre, ce qui laisse supposer qu'elles sont déterminées par un même locus. Dans ce cas, les 3 bandes trouvées chez chacun des individus peuvent s'expliquer par des phénomènes posttranscriptionnels intervenant, soit séquentiellement dans le temps, soit dans différents tissus. Des écarts de migration constatés pour ce locus et reproduits dans les figures 1 et 2 , on déduit qu'il existe au moins 3 allèles dans cette population. Si on accepte l'hypothèse de 3 allèles pour ces 20 clones, leur fréquence dans la population d'origine se répartis- 
TABLEAU 1

Techniques d'électrophorèse.

Electrophoresis techniques.

\begin{tabular}{|c|c|c|c|}
\hline Enzymes & Conditions d'extraction & Type de gel et tampon & Conditions de révélation \\
\hline Estérase & $\begin{array}{l}\text { Broyage de chaque puceron dans } \\
25 \mu \mathrm{l} \text { de sucrose } 17 \mathrm{p} \text {. } 100 \text { - Centrifu- } \\
\text { gations } 30 \mathrm{mn} \text { à } 40000 \mathrm{~g} \text {, puis } 20 \mathrm{mn} \text { à } \\
15000 \mathrm{~g}\end{array}$ & $\begin{array}{l}\text { Gel «spacer» sur gel homogène } \\
7 \mathrm{p} .100 \text { d'acrylamide, pH } 9,3 \text {, par } \\
\text { Tris } \mathrm{HCl} \text { - Plaque de } 400 \mu \text {. Tampon } \\
\text { cuve : Tris glycine, pH } 8,3,0,05 \mathrm{M}\end{array}$ & $\begin{array}{l}\text { Pour } 25 \mathrm{ml} \text { de tampon phosphate } \\
0,1 \mathrm{M} \text {, pH } 7,2,15 \mathrm{mg} \text { fast blue } \mathrm{RR} \text { et } \\
20 \mathrm{mg} \text { de naphthyl acétate }\end{array}$ \\
\hline $\begin{array}{l}\text { Malate déshydrogé- } \\
\text { nases }\end{array}$ & $\begin{array}{l}\text { Broyage de chaque puceron dans } \\
25 \mathrm{\mu l} \text { de Tris } \mathrm{HCl} \text {, pH } 8-0,1 \mathrm{M} \text { à } \\
17 \mathrm{p} .100 \text { de sucrose et } 0,5 \mathrm{p} .1000 \text { de } \\
\text { Triton } \times 100\end{array}$ & $\begin{array}{l}\text { Gel "spacer» sur gel homogène } \\
7 \text { p. } 100 \text { d'acrylamide, pH } 8,9 \text {, avec } \\
\text { Tris } \mathrm{HCl} \text { - Plaque de } 400 \mu \text { - Tampon } \\
\text { cuve : Tris glycine, pH } 8,3,0,05 \mathrm{M}\end{array}$ & $\begin{array}{l}\text { Pour } 25 \mathrm{ml} \text { de tampon Tris } \mathrm{HCl} \text {, } \\
0,5 \mathrm{M}, \mathrm{pH} 7,1-\mathrm{Na}_{2} \mathrm{CO}_{3} \text { et acide mali- } \\
\text { que }+(12,5 \mathrm{mg} \mathrm{NAD}, 7,5 \mathrm{mg} \mathrm{MTT} \text {, } \\
0,5 \mathrm{mg} \text { PMS })\end{array}$ \\
\hline \multirow[t]{2}{*}{ Phosphatases } & $\begin{array}{l}\text { Broyage du puceron dans } 25 \mu \mathrm{l} \text { de } \\
\text { sucrose à } 17 \mathrm{p} .100 \text { - Centrifugations } \\
30 \mathrm{mn} \text { à } 40000 \mathrm{~g} \text {, puis } 20 \mathrm{mn} \text { à } \\
15000 \mathrm{~g}\end{array}$ & $\begin{array}{l}\text { Gel «spacer» sur gel homogène } \\
7 \mathrm{p} .100 \text { d'acrylamide, pH } 8,7 \text {, avec } \\
\text { Tris } \mathrm{HCl} \text { - Plaque de } 400 \mu \text { - Tampon } \\
\text { cuve : Tris glycine, pH } 8,3,0,05 \mathrm{M} \text {. }\end{array}$ & $\begin{array}{l}\text { pH ac. : } 100 \mathrm{ml} \text { tampon acétate, } \\
0,05 \mathrm{M} \mathrm{pH} 5,100 \mathrm{mg} \text { de naphthyl } \\
\text { phosphate et } 50 \mathrm{mg} \mathrm{de} \mathrm{fast} \mathrm{garnet} \\
\text { GBC salt }\end{array}$ \\
\hline & & $\begin{array}{l}\text { Isofocalisation (Dépôt côté basique) - } \\
\mathrm{NaOH}, 1 \mathrm{~N} \mathrm{PO}_{3} \mathrm{H}_{4} 1 \mathrm{~N}-\mathrm{Ampho-} \\
\text { lithes 5-8 et } 7-9-\text { Plaques de } 100 \mu\end{array}$ & \\
\hline Glycosidases & $\begin{array}{l}\text { Broyage des pucerons dans } 0,5 \mathrm{ml} \text { de } \\
\text { sucrose à } 17 \mathrm{p} .100-\text { Triton X } 100 \\
0,5 \mathrm{p} .1000 \text { - PMSF } 0,035 \mathrm{p} .100- \\
2 \text { centrifugations à } 40000 \mathrm{~g} \text { et } 1 \text { cen- } \\
\text { trifugation (airfuge Beckmann) à } \\
100000 \mathrm{~g}\end{array}$ & $\begin{array}{l}\text { Gel à gradient } 2,30 \mathrm{p.} 100, \mathrm{pH} 8,3, \\
\text { avec Tris borate - Tampon cuve : Tris- } \\
\text { glycine, pH } 8,3,0,05 \mathrm{M} \text { - Migration : } \\
18 \mathrm{~h} .\end{array}$ & $\begin{array}{l}\text { Tampon acétate } 0,1 \mathrm{M} \text {, pH } 5 \text { - fast } \\
\text { blue } \beta \mathrm{B} \text { et substrat selon enzyme } \\
\text { choisi. Dissolution dans DMSO pour } \\
2 \beta \mathrm{D} \text { galactosidoxy naphtol AS-LC } \\
\text { et pour } 6 \text { bromo } 2 \text { naphtyl } \beta \text { D glucu- } \\
\text { ronide }\end{array}$ \\
\hline
\end{tabular}

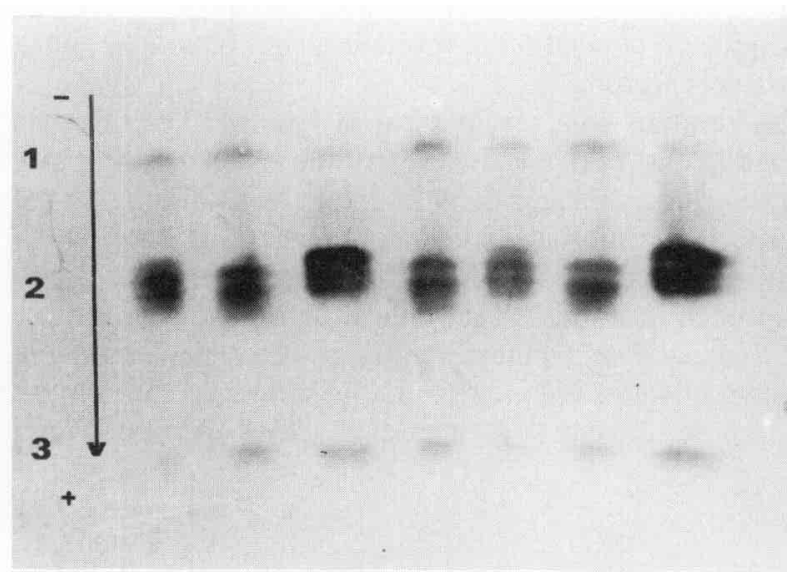

Figure 1

Isoestérases chez Acyrthosiphon pisum observées chez des individus "clonés", à partir d'une population naturelle. On notera la variabilité allélique au locus 2.

Isoesterases of Acyrthosiphon pisum observed on clones raised from a wild population. Note the allelic variability at locus 2 .

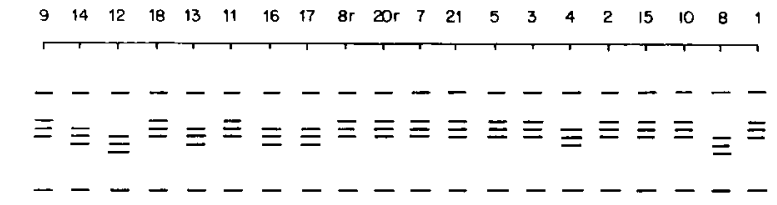

Figure 2

Isoesterases chez Acyrthosiphon pisum. Le locus 2 comprend 3 bandes non alléliques se déplaçant de façon concomitante en fonction des 3 allèles représentés dans la population.

Isoesterases of Acyrthosiphon pisum. Locus 2 showed a triple nonallelic band moving together according to the 3 alleles present in the population. sait ainsi : allèle lent $=13$, allèle médian $=5$, allèle rapide $=2$.

Enfin, par rapport à ce groupe polymorphe d'estérases, il existe 2 autres bandes importantes situées de part et d'autre du "groupe 2 »; une migrant moins, l'autre davantage. Elles appartiennent vraisemblablement à 2 loci distincts. II est possible qu'un ou plusieurs de ces loci puissent jouer un rôle dans la détoxification des organophosphorés de la même façon que cela a été démontré pour $M$. persicae et évoqué pour $A$. fabae par BERANEK (1974). Une étude plus axée sur ces enzymes permettrait de préciser ce point.

\section{B. La malate déshydrogénase}

La malate déshydrogénase se manifeste sous forme de 2 ensembles principaux. Un $1^{\text {er }}$ difficile à analyser, un $2^{\mathrm{e}}$ qui migre plus et comprend distinctement 3 bandes, également espacées. LA MDH étant un enzyme dimère, la bande médiane peut être une bande hybride, la situation de ce locus correspondrait à une hétérozygotie permanente comportant 2 allozymes. Le $1^{\text {cr }}$ ensemble à migration lente paraît composite et une certaine variabilité n'est pas à exclure. On est ici gêné par l'impureté du matériel qui ne donne pas de bandes bien définies; de plus, la migration de la $\mathrm{MDH}_{1}$ est trop faible pour que l'on puisse porter un jugement précis sur son caractère polymorphe ou non. On notera que SUOMALAINEN et al. (1980) et TOMIUK \& WÖHRMANN (1980), opérant sur gels d'amidon, signalent 2 loci dont un, $\mathrm{MDH}_{1}$, polymorphe. SIMON et al. (1982) n'ont pas trouvé de polymorphisme à ce locus, mais ils ont opéré sur d'autres populations et dans des conditions différentes d'électrophorèse. Les gels de polyacrylamide à 7 p. 100 , pH 8,9, utilisés ici, séparent donc les bandes du $2^{\mathrm{e}}$ locus, mais discriminent mal le premier. 


\section{Phosphatase alcaline et acide}

Dans les conditions d'électrophorèse décrites, on observe une bande migrant à $1,2 \mathrm{~cm}$ pour une migration du bleu de bromophénol de $6 \mathrm{~cm}$. Cet enzyme est plus actif à $\mathrm{pH}$ alcalin, mais présente également une forte activité à $\mathrm{pH}$ acide.

A pH acide, après prétrempage du gel dans l'acide borique à $\mathrm{pH} \mathrm{5}$, on révèle une phosphatase acide à migration plus rapide, se traduisant par une bande relativement faible et plutôt de couleur orangée. Aucun polymorphisme n'a été observé pour ces 2 phosphatases pour la population considérée (fig. 3a).

\section{Les glycosidases}

Quatre groupes d'enzymes ont été étudiés sur gel en utilisant les 10 substrats qui figurent au tableau 2.

La quantité d'enzymes présente dans un individu étant très faible (à part 1 ou 2 exceptions), leur mise en évidence n'a été possible, même sur microgels, que sur des extraits obtenus à partir d'une centaine d'individus. Tous les lots examinés et tous les clones ont donné les mêmes enzymogrammes pour chacun des enzymes.

Si les enzymes examinés ont, d'une manière générale, été choisis pour des commodités de révélation, cela n'a pas été
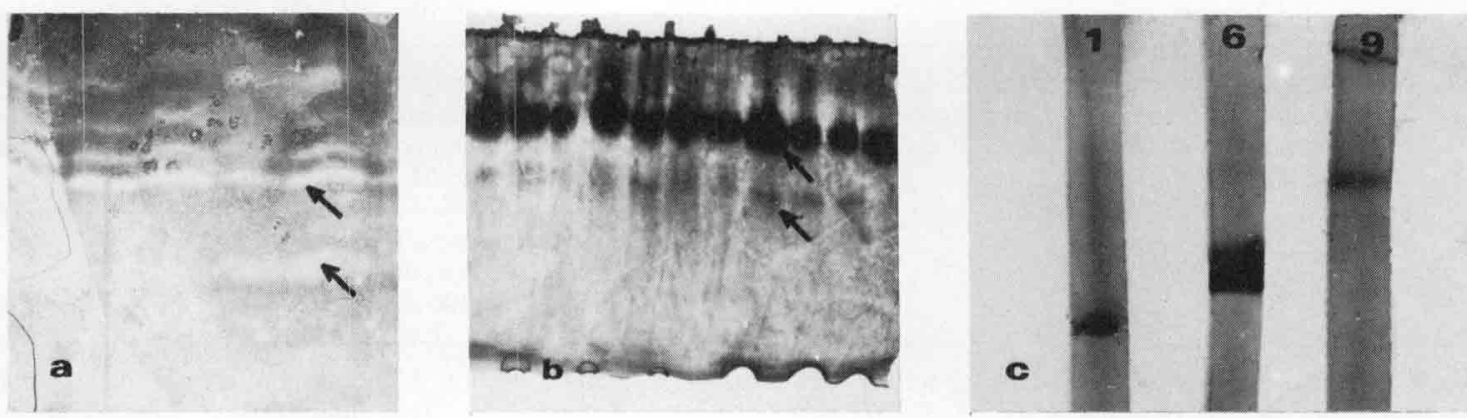

Figure 3

$a$ et $b$ - Phosphatases acides et basiques chez Acyrthosiphon pisum. $a$ : électrofocalisation gamme 5-9; b: gel acryl. 7 p. 100, pH 8,7; $c$ : glycosidases (1: $\beta$-galactosidase ; $6: N$-naphihyl-glucosaminidase; 9 : glucuronidase).

$a$ and $b$ - Acid and basic phosphatases in Acyrthosiphon pisum. $a$ : isofocusing, ampholine range $5-9 ; b: 7 \%$ acrylamide gel, pH 8.7;

$c:$ glycosidases (1: $\beta$-galactosidase; $6: N$-naphthyl-glucosaminidase ; 9 : glucuronidase).

En isofocalisation, on retrouve 2 ensembles de phosphatases : côté basique, une bande rouge orangée assez faible et vers les $\mathrm{pH}$ acides une série de bandes ( 4 bien discernables) s'échelonnant à l'arrière d'une bande principale foncée vers les pH acides. Bien qu'il n'ait été procédé à aucune double dimension, en vue d'établir une corrélation avec les 2 loci obtenus avec le système normal d'électrophorèse, on peut penser que l'on retrouve ici les 2 entités précédentes. Le plus grand nombre des bandes foncées en isofocalisation traduirait des états isoélectriques différents de la même phosphatase, très abondante chez $A$. pisum. Ici encore aucun polymorphisme n'a été décelé dans la population examinéc (fig. 3b). tout à fait le cas pour les glycosidases, rarement utilisées en génétique de population et révélées sur gel pour la $1^{\text {re }}$ fois à partir de puceron.

Les résultats sont résumés par la figure 4. Les substrats utilisés n'ont permis de révéler que quelques enzymes pouvant être impliqués dans les interactions avec la plante et déterminer ainsi de possibles biotypes. Quant à la Nacétyl-glucosaminidase, sa relative abondance est peut-être à mettre en relation avec la synthèse de la chitine ou la mue.

L' $\alpha$-glucosidase donne 6 bandes isoenzymatiques avec le substrat $n^{\circ} 4$ et 4 avec le $n^{\circ} 5$. La différence se situe sur un groupe de 2 bandes à migration plus importante qui

TABLEAU 2

Substrats utilisés pour révéler les glycosidases. Les résultats sont donnés par la figure 4. Substrates used to detect glycosidases. Results are given in fig. 4.

\begin{tabular}{|c|c|c|}
\hline Enzymes révélés & $\begin{array}{l}\text { No Référence } \\
\text { voir figure } 4\end{array}$ & Substrats \\
\hline Galactosidases & $\begin{array}{l}1 \\
2 \\
7\end{array}$ & $\begin{array}{l}6 \text { bromo } 2 \text { naphthyl } \beta \text { D galactopyranoside } \\
6 \text { bromo } 2 \text { naphthyl } \alpha \text { D galactopyranoside } \\
2 \beta \text { D galactosidoxy naphthol AS-LC }\end{array}$ \\
\hline Glucosidases & $\begin{array}{l}3 \\
4 \\
5\end{array}$ & $\begin{array}{l}6 \text { bromo } 2 \text { naphthyl } \beta \text { D glucoside } \\
6 \text { bromo } 2 \text { naphthyl } \alpha \mathrm{D} \text { glucoside } \\
\beta \text { naphthyl } \alpha \mathrm{D} \text { glucopyranoside }\end{array}$ \\
\hline Glucuronidases & $\begin{array}{r}8 \\
9 \\
10\end{array}$ & $\begin{array}{l}\text { Naphthol AS B1 } \beta D \text { glucuronic acid } \\
6 \text { bromo } 2 \text { naphthyl } \beta \text { D glucuronide } \\
\alpha \text { naphthyl } \beta \text { D glucelronic acid }\end{array}$ \\
\hline Glucosaminidases & 6 & $N$ acetyl $\beta$ glucosamine naphthol \\
\hline
\end{tabular}




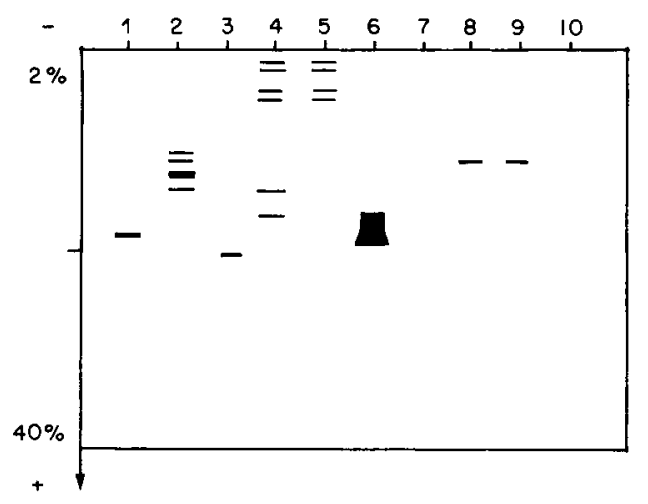

Figure 4

Localisation des glucosidases d'Acyrthosiphon pisum sur gel à gradient 2, 40 p. $100: 1,2,3, \ldots$ correspondent aux différents substrats (voir tableau 2).

Localization of the glycosidases from Acyrthosiphon pisum on gradient gel $2,40 \%: 1,2,3, \ldots$ correspond to the different substrates (see table 2).

n'apparaissent qu'avec le $1^{\text {er }}$ substrat, sans doute à cause d'une meilleure affinité puisque l'échantillon est identique. Le groupe commun composé de 4 bandes, en 2 paires, migre très peu. Si on prend en compte la composition du gel à gradient, cela correspond à des poids moléculaires élevés qui ne peuvent se comprendre que par l'existence de molécules polymérisées. Il en est peut-être de même pour d'autres glycosidases.

Les $\beta$-glucosidase, $\beta$-galactosidase et $\beta$-glucuronidase se manifestent sous forme d'une bande et l' $\alpha$-galactosidase d'un groupe de 4 bandes.

Il n'est pas certain, étant donné que ces profils isoenzymatiques correspondent à une somme d'individus, que l'on retrouverait les mêmes pour chacun des individus (notamment pour $\alpha$-glucosidase et $\alpha$-galactosidase où plusieurs bandes sont présentes). De plus, il est impossible de tirer des informations sur le degré de polymorphisme pour ces enzymes, car les gels à gradient éliminent " l'effet charge ", facteur courant de la variabilité des protéines, bien avant les questions de masse et de conformation qui n'apparaissent qu'après des modifications plus importantes.

L'étude de la variabilité biochimique de ces molécules, dont peut-être certaines sont engagées dans l'interaction avec l'hôte, mérite un nouvel effort technologique.

\section{DISCUSSION ET CONCLUSION}

Les résultats obtenus confirment qu'il existe d'une façon générale peu de polymorphisme biochimique chez les puce- rons. Cela avait déjà été souligné par MAY \& HOLBROOK (1978), SuOMALAINEN et al. (1980), TOMIUK \& WÖHRMANN (1980), ... Les pucerons ne constituent pas une exception ; on signale de plus en plus d'organismes présentant de faibles variabilités lorsque leurs protéines sont analysées par électrophorèse, par exemple les nématodes Meloidogyne, qui ont des polymorphismes biochimiques pratiquement nuls (DAlmasso \& Bergé, 1978). Dans le cas présent, seule une estérase a présenté une variation évidente; les autres loci, à quelques réserves près, sont apparus monomorphes. Mais on peut remarquer que les examens ont été réalisés à partir de lots restreints d'individus provenant d'un même lieu, ce qui a pu sérieusement limiter la diversité. Par ailleurs on connaissait chez $A$. pisum des différences dans le comportement alimentaire et on pouvait espérer trouver quelque variabilité pour les glucosidases, galactosidases ou glucuronidases. Or, on remarque que les bandes sont relativement constantes quant à leur migration ; cependant, la technique en gel à gradient élimine les variations associées à la charge des protéines. De plus, l'utilisation dans un même échantillon de plusieurs individus interdit toute analyse quantitative individuelle et on sait que des changements génétiques subtils peuvent intervenir dans la descendance par différents systèmes, telle que l'amplification génétique à un locus (BUNTING \& VAN EMDEN, 1980).

Ainsi, à la faveur de méthodologies permettant l'évaluation des diversités génétiques des populations sur les isoenzymes, un nouveau pas a été fait et une autre façon d'apprécier le contexte des relations est apparue : il semble cependant que cette nouvelle manière, même si elle marque un tournant intéressant, sera elle-même insuffisante, car la diversité fonctionnelle des organismes n'est pas entièrement reflétée dans celle de leur polymorphisme protéinique, tel qu'il est souvent perçu en électrophorèse. Interviennent aussi les phénomènes d'amplification, d'induction et de régulation sur lesquels nous sommes encore mal renseignés et qui sont moins perceptibles. Pour ce qui concerne la nutrition du puceron et la variabilité de son comportement vis-à-vis de telle ou telle plante, il conviendrait d'analyser les enzymes digestifs secrétés par des techniques de microélectrophorèse après marquage isotopique ou en recourant aux très sensibles colorations à l'argent, sachant bien que la solution ne résidera pas entièrement dans ces seules protéines, mais cela constituerait déjà un pas important vers une compréhension plus globale de ces phénomènes.

Reçu le 9 février 1983. Accepté le 16 mai 1983.

\section{RÉFÉRENCES BIBLIOGRAPHIQUES}

Baker J. P., 1977. Assessment of the potential for and development of organophosphorus resistance in field population of Myzus persicae. Ann. appl. Biol., 86, 1-9.

Beranek A. P., 1974. Esterase variation and organophosphate resistance in populations of Aphis fabae and Myzus persicae. Entomol. exp. appl., 17, 129-142.

Bournoville R., 1977. Etude de quelques relations entre le végétal (espèce, variété, stàde phénologique) et le puceron du pois Acyrthosiphon pisum Harris (Homoptera : Aphididae). Ann. Zool. Ecol. anim., 9, 87-98.

Bunting S., Van Emden H. F., 1980. Rapid response to selection for increased esterase activity on small populations of an apomictic clone of Myzus persicae. Nature, 285, 502-503.

Dalmasso A., Bergé J. B., 1978. Molecular polymorphism and phylogenetic relationship in some Meloidogyne ssp. : Application to the taxonomy of Meloidogyne. J. Nematol., 10, 323-332.
Lapchin L., Pintureau B., Boll R., 1981. Mise en évidence par électrophorèse d'une modification enzymatique des œufs de Myzus persicae Sulz., au moment de la levée de diapause. Agronomie, 1, 519.

Maurer H. R., 1971. Disc electrophoresis and related techniques of polyacrylamide gel electrophoresis. W de Gruyter Ed., $222 \mathrm{p}$.

May B., Holbrook F. R., 1978. Absence of genetic variability in the green pcach aphid, Myzus persicae (Hemiptera: Aphididae). Ann. Entomol. Soc. Am., 71, 809-811.

Sawicki R. M., Devonshire A. L., Rice A. D., Moores G. D., Petzing S. M., Cameron A., 1978. The detection and distribution of organophosphorus and carbamate insecticide-resistant Myzus persicae Sulz. in Britain in 1976. Pestic. Sci., 9, 189-201.

Shaw C. R., Prasad R., 1969. Starch gel electrophoresis of enzymes. A compilation of recipes. Biochem. Genet., 4, 297-320. 
Simon J. P., Parent M. A., Auclair J. L., 1982. Isozyme analysis of biotypes and field populations of the pea aphid, Acyrthosiphon pisum. Entomol. exp. appl., 32, 186-192.

Singh S. M., Cunningham T. K., 1981. Morphological and genetic differentiation in aphids (Aphididae). Can. Entomol, 113, 539-550. Sudderuddin K. I., 1972. An electrophoretic study of some hydrolases from an OP'susceptible and OP-resistant strain of the green peach aphid, Myzus persicae Sulz. Comp. Biochem. Physiol., 44 B, 923-929.

Suomalainen E., Saura A., Lokki J., Teeri T., 1980. Genetic polymorphism and evolution in parthenogenetic animals. part 9. Absence of variation within parthenogenetic aphid clones. Theor. appl. Genet., 57, 129-132.
Takada H., 1979. Characteristics of forms of Myzus persicae Sulz. (Homoptera: Aphididae) distinguished by colour and esterase differences and their occurence in populations on different host plant in Japan. Appl. Entomol. Zool., 14, 370-375.

Tomiuk J., Wöhrmann K., 1980. Enzyme variability in populations of aphids. Theor. appl. Genet., 57, 125-127.

Wool D., Bunting S., Van Emden H. F., 1978. Electrophoretic study of genetic variation in british Myzus persicae Sulz. (Hemiptera: Aphididae). Biochem. Genet, 16, 987-1006. 